\title{
Spectral Measurements of the Second Harmonic of the SASE FEL Radiation at $\mathrm{APS}^{\curlyvee}$
}

\author{
V. Sajaev*, Z. Huang \\ Advanced Photon Source, Argonne National Laboratory \\ Argonne, IL 60439
}

\begin{abstract}
We present the z-dependent spectral measurement results for the second harmonic of the SASE FEL radiation before and after saturation. The measurements were performed at the Advanced Photon Source FEL with the fundamental wavelength of $530 \mathrm{~nm}$. The spectral properties of the second harmonic are compared with those of the fundamental and with the theoretical expectations.
\end{abstract}

PACS codes: 41.60.Cr

Keywords: Free-electron laser; Self-amplified spontaneous emission; Spectrum

\section{Introduction}

The generation of harmonics through a nonlinear mechanism driven by bunching at the fundamental has stimulated interest as a way to enhance and extend an X-ray free-electron laser (FEL) facility. The microbunching at the fundamental that occurs during the FEL process has a spiky shape near saturation. When Fourier decomposed, it shows significant harmonic content. These nonlinear

\footnotetext{
$\diamond$ Work supported by the US Department of Energy, Office of Basic Energy Sciences, under Contract No. W-31-309-ENG-38.

* Corresponding author.

E-mail address: sajaev@aps.anl.gov (V.Sajaev)
}

harmonics are driven by the fundamental and experience gain to saturation with gain lengths that vary as the inverse of the their harmonic number [14].

There are a number of papers describing recent measurements of nonlinear harmonics. These include the high-gain harmonic generation experiment at Brookhaven National Laboratory (BNL) [5] and harmonic spectral measurements at Osaka University, Japan [6] and at the VISA experiment at BNL [7].

This paper extends the first analysis of the 
spectrum of the second harmonic measured at the low-energy undulator test line (LEUTL) at the Advanced Photon Source (APS) and presented earlier in Ref. [8]. In this paper we present the z-dependent spectral measurement results and their analysis for the second harmonic of the SASE FEL before and after saturation.

\section{Measurements}

The low-energy undulator test line at the APS is designed to conduct experiments on the SASE FEL process in the visible and ultraviolet wavelengths. It was built as an extension of the existing APS linac and includes a photocathode rf gun, a linear accelerator with a bunch compressor, an electron beam transport line, and an undulator system. A detailed description of the LEUTL and its various component systems can be found in Ref. [9].

All spectral measurements reported in this paper have been done with a high-resolution spectrometer located at the downstream end of the undulator line in the end-station room. The undulator system consists of eight identical 2.4-m-long undulators; after each undulator there is a visible light diagnostics (VLD) station. A mirror at each diagnostic station can direct the SASE light towards the spectrometer through a hole in the shielding wall, thus allowing one to measure spectral characteristics of the SASE light at different longitudinal locations along the undulator line. The spectrometer has been calibrated with hollow cathode discharge lamps for both fundamental and second harmonic wavelengths. A detailed description of the spectrometer can be found in Ref. [10].

The electron beam parameters measured immediately after the spectrum measurements are presented in Table 1. The gain scan is a routine measurement and is done before any measurements. Figure 1 shows the experimental fundamental 530$\mathrm{nm}$ gain scan results and those obtained from the GINGER [11] simulations corresponding to the beam parameters in Table 1. The close correspondence between the measured and simulated gain curves ensures us that the beam parameters used in the simulations are close to the real experiment.
Table 1. Electron beam parameters

\begin{tabular}{|l|c|}
\hline Beam energy $(\mathrm{MeV})$ & 217 \\
\hline Beam charge $(\mathrm{pC})$ & 470 \\
\hline Emittance $\mathrm{x}(\mu \mathrm{m})$ & 7.2 \\
\hline Emittance y $(\mu \mathrm{m})$ & 4.6 \\
\hline Rms bunch length $(\mathrm{ps})$ & 0.5 \\
\hline Rms energy spread $(\mathrm{MeV})$ & 0.6 \\
\hline
\end{tabular}

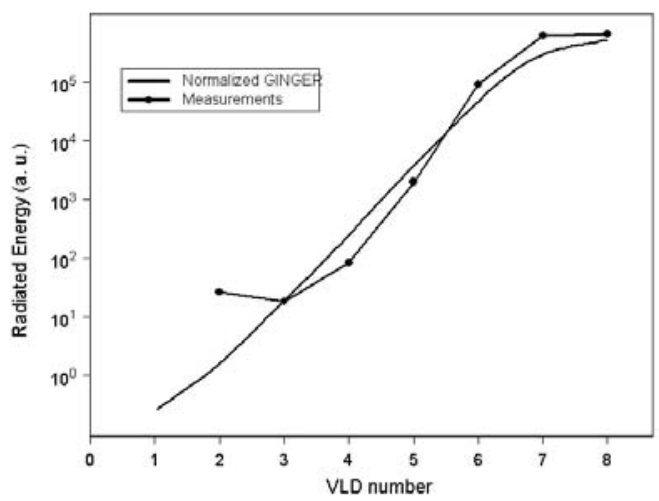

Figure 1. Measured fundamental energy compared with the simulated counterpart.

Since the second harmonic radiation starts growing later than the fundamental and grows faster, we were able to detect the spectrum of the second harmonic only after undulator 6 and beyond. As one can see from Figure 1, saturation also occurs after undulator 6 . That gives us only one measurement point in the exponential gain regime and two points after saturation.

Figure 2 shows typical single-shot spectra taken after undulators 6,7 , and 8 . Note that the number of spikes increases from VLD station 6 to station 8 . This is consistent with the gain scan showing saturation of the fundamental after undulator number 6 . 

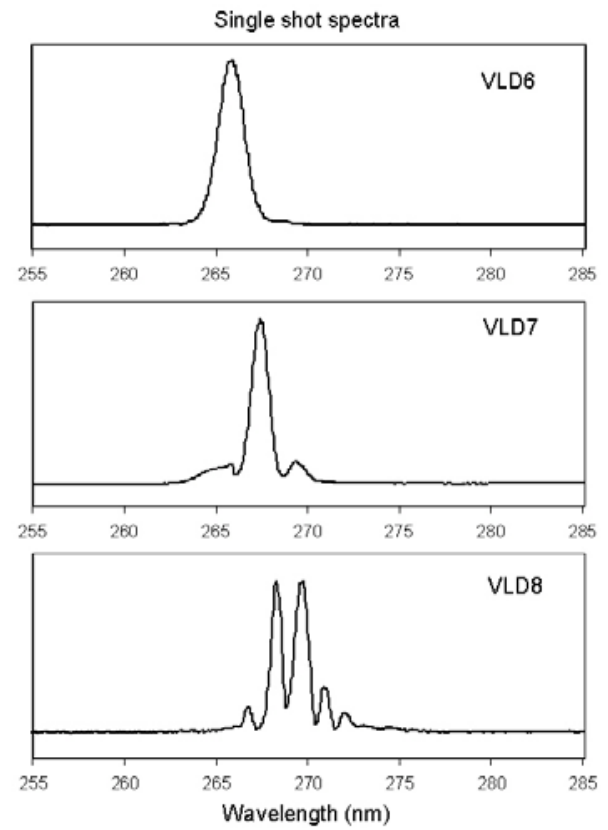

Figure 2. Typical single-shot spectra of the second harmonic. One can clearly see the increase of the number of spikes from station 6 to 8 .

The spectrum width of the second harmonic in the exponential gain regime can be estimated analytically. In the exponential growth regime, the $n$ th order nonlinear harmonic field scales as

$$
E_{n}(t) \propto E_{1}(t)^{n} .
$$

Within one coherent spike, one can write

$$
E_{1}(t) \sim \exp \left(-\frac{t^{2}}{4 \sigma_{t}^{2}}\right),
$$

where $\sigma_{t}$ is the coherence time for the fundamental field $\mathrm{E}_{1}$. Thus, the coherence time of the $n$-th nonlinear harmonic is

$$
\sigma_{t n}=\frac{\sigma_{t}}{\sqrt{n}} .
$$

After applying the Fourier transform theorem, the spectral bandwidth is given by

$$
\left(\frac{\sigma_{\omega}}{\omega}\right)_{n}=\left(\frac{\sigma_{\lambda}}{\lambda}\right)_{n}=\frac{\lambda_{n}}{4 \pi \sigma_{t n}},
$$

where $\lambda_{n}=\lambda / n$. Therefore, for the $n$-th harmonic, the relative bandwidth is narrowed by $\sqrt{n}$.
Thus, we expect the spectrum width of the second harmonic to be narrower than the fundamental by roughly a factor of 1.4. To compare this ratio with the experiment, Figure 3 shows the measured average rms spectrum width for both the fundamental and second harmonic and the simulations (only for fundamental). The simulations demonstrate very good approximation of the measured spectrum width. In the exponential gain regime (up to station 6) the difference between the measured and calculated spectrum width is less than $10 \%$. After the saturation this difference increases but stays below $20 \%$. The measured spectrum width of the second harmonic at the exponential growth regime (at VLD 6) is narrower than the fundamental by the expected factor of 1.4. Narrowing of the harmonic spectrum width with increasing harmonic order has also been observed in Ref. [7]. After saturation, both the fundamental and the second harmonic spectral widths are expected to increase due to the appearance of sidebands. However, the GINGER simulation does not directly compute the harmonic radiation that would enable comparison with the measured harmonic spectral width.

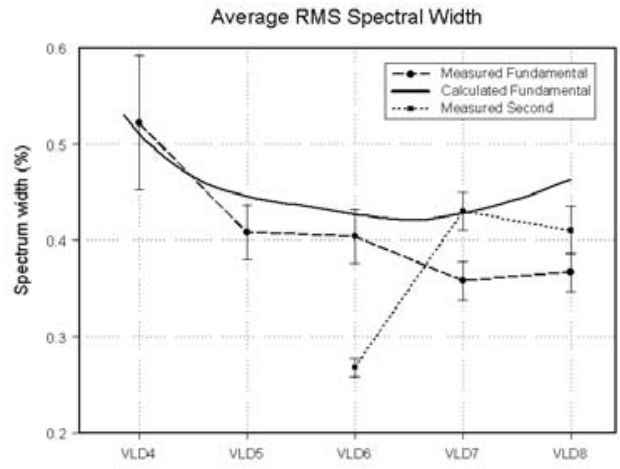

Figure 3. The average rms spectral width of the fundamental and second harmonic compared with the simulated counterpart for the fundamental.

From Figure 2 one can see that the radiation spectrum after undulator 6 has only one spike while two other spectra show two or more spikes. The spectral shape was analysed in terms of number of spikes and their width.

For spontaneous radiation on the fundamental wavelength the width of the spectral spikes corresponds to the reciprocal of the bunch length. 
Since the electron bunch profile is not flat and because the gain depends on the local electron bunch current, radiation emitted by different parts of the beam experiences different gain. One can consider it as a decrease in the effective bunch length, because the radiation intensity in the most central intense part of the beam grows faster than in the rest of the beam. Therefore the spike width will increase during the exponential gain regime. At saturation, other parts of the beam with lower local current will also come to saturation. This will result in an increase in the effective bunch length and a corresponding decrease in the spike width. These arguments are true for the fundamental radiation, but later we will show that the second harmonic behaviour follows the fundamental one.

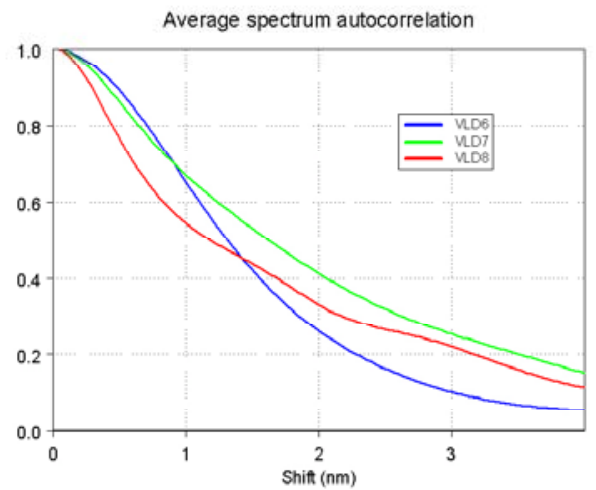

Figure 4. Average spectrum autocorrelation of the second harmonic.

The spike width can be extracted from an autocorrelation of the spectrum. Figure 4 shows the autocorrelation of the measured second harmonic spectrum averaged over many shots. The average width of the single spike is related to the "near-term" width of the autocorrelation (less than $1 \mathrm{~nm}$ shift) while the overall spectrum width is related to the "long-term" autocorrelation width. According to that, the curve corresponding to VLD 6 shows wide spikes and the narrowest overall spectrum width. Conversely, the VLD 8 curve shows the narrowest single spikes. This means that for the part of the gain process that we can observe, the behaviour of the second harmonic spectrum follows the behaviour of the fundamental. Additionally, the average number of spikes increases from 1.2 for VLD 6 to 1.7 for VLD 7 to 2.3 for VLD 8 .

\section{Conclusion}

The z-dependence of the spectrum of the second harmonic of SASE FEL radiation has been measured. Despite the fact that the individual second harmonic spectra may not exactly replicate the fundamental [10], for the part of the gain process that we could observe, the average behaviour of the second harmonic spectrum follows the behaviour of the fundamental. It shows an increase of the spectrum width, the appearance of second harmonic sidebands, and spike narrowing after saturation. The measured spectrum width corresponds well to the analytical estimate for the second harmonic in the exponential gain regime. Unfortunately, at this point, there are no codes that can calculate the spectrum of the harmonics, so we cannot compare the measured spectral shape with the simulations.

The authors would like to thank S. Milton and J. Lewellen for continuous support. One of authours (ZH) would like to thank K.-J. Kim and W. Fawley for useful discussions.

\section{References}

[1] R. Bonifacio et al., Nucl. Instrum. and Methods. A 293 (1990) 627.

[2] H.P. Freund, S.G. Biedron, S.V. Milton, IEEE J. Quantum Electron, 36 (2000) 275.

[3] Z. Huang, K.-J. Kim, Phys. Rev. E 62 (2000) 7295.

[4] Z. Huang, K.-J. Kim, Nucl. Instrum. and Methods. A 475 (2001) 112.

[5] A. Doyuran et al., Phys. Rev. Lett. 86 (2001) 5902.

[6] R. Kato et al., Nucl. Instrum. and Methods. A 483 (2002).

[7] A. Tremain et al., Phys. Rev. Lett. 88 (2002).

[8] S.G. Biedron et al., Nucl. Instrum. and Methods. A 483 (2002) 94.

[9] S.V. Milton et al., Science 292 (2001) 2037.

[10] V. Sajaev et al., to appear in Nucl. Instrum. and Methods.

[11] W.M. Fawley, A user manual for GINGER and its postprocessor XPLOTGIN, LBNL-49625, 2002. 